\title{
代谢途径的进化
}

\author{
孙桂玲 ${ }^{1,2}$, 江永海 ${ }^{1,2}$, 文建凡 ${ }^{1, *}$ \\ (1. 中国科学院昆明动物研究所 遗传资源与进化国家重点实验室, 云南 昆明 650223；2. 中国科学院研究生院, 北京 100049)
}

摘要：生物的新陈代谢是通过一系列的代谢途径来实现的。这些调节精妙、相互协作的代谢途径是如何进化 形成的一直是一个引人入胜的重要问题。自 1945 年有关该问题的第一个假说——“逆向进化假说”提出以来, 迄 今已发展出七种假说，包括“逆向进化假说”、“半酶理论”、“向前发展模型”、“酶的招募假说”、“多功能酶特化假 说”、“整个代谢途径的复制假说”和“从头创造假说”。其中最受关注的是“逆向进化假说”和“酶的招募假说”，而最 近提出的“多功能酶特化假说”由于有较好的理论基础和实验证据支持，也逐渐引起人们的关注。本文对这些假说 逐一作了概述,并结合作者的相关研究工作,对该领域的研究现状和发展趋势进行了分析讨论和展望。

关键词：代谢途径；进化；假说

中图分类号: Q591 文献标识码: A 文章编号：0254-5853(2008)04-0459-06

\section{Evolution of Metabolic Pathways}

\author{
SUN Gui-ling ${ }^{1,2}$, JIANG Yong-hai ${ }^{1,2}$, WEN Jian-fan ${ }^{1 *}$ \\ (1. State Key Laboratory of Genetic Resources and Evolution, Kunming Institute of Zoology, the Chinese Academy of Sciences, Kunming 650223, China; \\ 2. Graduate School of the Chinese Academy of Sciences, Beijing 100049, China)
}

\begin{abstract}
The metabolism of organisms is carried out through a series of metabolic pathways. How these ingeniously regulated and collaborated metabolic pathways evolved has been a fascinating and important question for some time. Since the 'retro-evolution hypothesis' was first put forward in 1945, there have been seven main hypotheses concerning the evolution of metabolic pathways, including 'retro-evolution of pathways, 'semi-enzymatic theory', 'forward development model', 'enzyme recruitment hypothesis', 'specialization of multifunctional enzyme', 'pathway duplication', and 'de novo invention of pathways'. Among them, the most popular hypotheses are 'retro-evolution' and 'enzyme recruitment', and recently greater attention has been paid to the 'specialization of multifunctional enzyme' hypothesis, due to its strong theoretical basis and experimental evidence. Here, all of the seven hypotheses were reviewed, and the development prospect of this field analyzed.
\end{abstract}

Key words: Metabolic pathway; Evolution; Hypothesis

生物的新陈代谢是由一系列的代谢途径具体来 完成的。正是由于这些代谢途径的本身的精妙调节 和相互间的协调一致，生命活动才得以有条不紊地 进行。因此代谢途径是如何起源、形成和进化发展 的问题, 一直是一个极为引人入胜的重要生物学问 题，但迄今仍悬而未决。经过前人不断的研究和探 索，目前有关代谢途径起源进化的假说有七种之 多，它们各有各的证据所支持，但又存在各自的不 足。下面我们对它们逐一进行简介和讨论, 并对该 领域的研究现状和发展趋势进行分析和展望。

\section{1 逆向进化假说、半酶理论和向前发展模型}

逆向进化（retro-evolution or retrograde）假说是 Horowitz 于 1945 年提出的, 又称阶梯模型 (stepwise model）(Horowitz，1945)。该假说提出的前提有： 1) 最早的生命体是异养的, 靠消耗生命起源前就 已经存在的有机物来进行复制；2）中间代谢物可 以在环境中稳定存在；3）有一个包含终产物和潜 在中间物的特殊化学环境。该假说的核心内容是: 基础合成途径的进化是阶梯式的，一段时间内只有 一次突变发生, 每步合成途径形成的方向与现今合

收稿日期：2008-03-31；接受日期：2008-06-10

基金项目：科技部 973 项目（2007CB815705); 国家自然科学基金（30021004，30623007); 云南省基金（2007C098M）

*通讯作者（Corresponding author）, E-mail: wenjf@mail.kiz.ac.cn 
成途径进行的方向相反。具体的进化历程如下：生 命起源早期原始细胞生活在具有生命起源前复合 物(物质 $\mathrm{A} 、 \mathrm{~B} 、 \mathrm{C} 、 \mathrm{D}$ 和 $\mathrm{E}$ 等)的原始海洋中, 细胞 可以直接从环境中获得物质 $\mathrm{A}$ 用于生命活动, 同时 环境中还存在前体物质 $\mathrm{B}$ 和 $\mathrm{C}$ [ 物质 $\mathrm{B}$ 和 $\mathrm{C}$ 在催化 剂（酶）的作用下可以合成为 $\mathrm{A}$ ]。当物质 $\mathrm{A}$ 被消 耗到影响细胞的进一步生长时, 出现具有酶 1

(Enz1) 可以利用 B 和 C 合成 A 的突变株, 并占 据选择优势; 当 $\mathrm{A}$ 在环境中消失后, 该突变株取代 原有细胞株。同理, 当因 B 消耗到一定程度时, 此 时, 出现具有酶 2(Enz2) 可以利用环境中的 D 和 $\mathrm{E}$ 来合成 $\mathrm{B}$ 的突变株, 并具有选择优势 (Horowitz, 1945; Rison et al, 2002)。由于 Enz2 要像 Enz1 一 样能够结合 B, 所以 Enz2 可能来自 Enz1 的基因复 制 (Light et al, 2004)。上述步骤的重复最终形成 现今的代谢途径 (Rison et al, 2002)。也就是说, 逆向进化机制是一种底物驱动的进化方式, 一旦含 有稳定代谢中间物的有机环境被破坏, 代谢途径的 这种进化方式也就停止了（Horowitz，1945）。

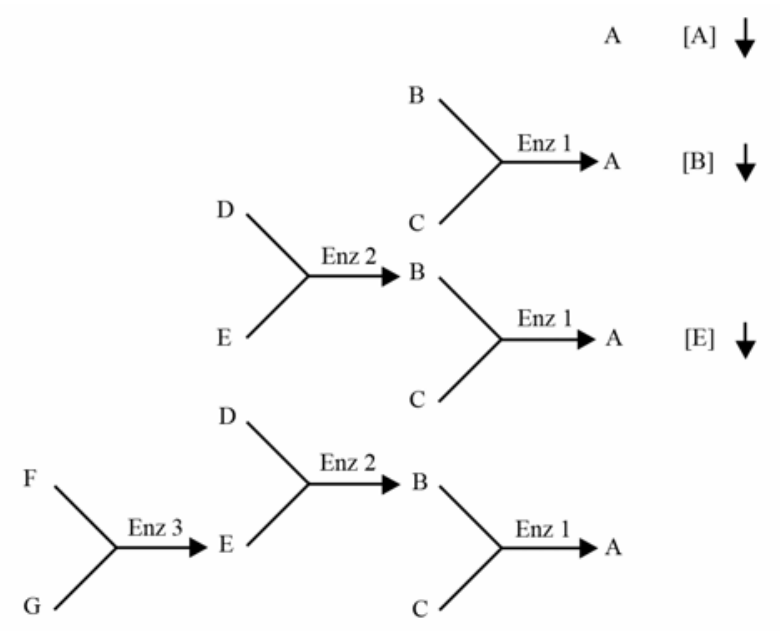

图 1 代谢途径的逆向进化假说 (Rison et al, 2002)

Fig. 1 Retro-evolution hypothesis of metabolic pathways (Rison et al, 2002)

向下的箭头表示物质浓度逐渐降低。

The arrow down indicates the concentration of the substances reduced gradually.

从上述可以看出, 逆向进化假说的优点在于可 以很好地解释为应答环境变化 (稳定代谢中间物的 减少或消失) 合成途径的快速进化（Horowitz, 1945 )。其缺陷是不能解释包含不稳定代谢中间物 的代谢途径的形成。此外, 该假说的另一个前提
一最早的生命体是异养的，虽然有很多的证据支 持，但仍然有些人认为最早的生命体是自养的

(Hartman, 1975)。

逆向进化假说的一个很好的例子是糖酵解途 径。糖酵解途径是细胞主要的代谢途径之一，同时 也是一个非常古老的代谢途径（Romano et al， 1996)。真核细胞一般具有完整的糖酵解途径, 但 很多真细菌只具有从磷酸甘油醛脱氢酶到丙酮酸 激酶的“主干部分”; 而在一些最原始并且进化最慢 的古菌中，糖酵解酶则更少，例如喏热酸菌

( thermoacidophiles) 只有催化最后两步反应的酶 (烯醇化酶和丙酮酸激酶), 于是有人认为糖酵解 途径的形成是从基部逐渐向上演化的

(Fothergill-Gilmore et al, 1993)。此外, 逆向进化 假说认为处于同一代谢途径且催化连续反应步骤 的酶是通过基因重复而来, 是同源的。对于这一点, Nahum \& Riley (2001) 在调查大肠杆菌序列相关的 蛋白群时发现, 一些处于同一代谢途径且催化连续 反应步骤的酶确实是同源的。例如, 甲硫氨酸合成 途径中的 MetB 和 MetC (Belfaiza et al, 1986), 色 氨酸合成途径中的 $\operatorname{trpC} 、 \operatorname{trp} A$ 和 $\operatorname{trpB}$ (Wilmanns et al，1991）以及组氨酸合成途径中的 HisF 和 HisA (Fani et al, 1995), 它们催化连续的反应步骤, 并 且都是同源的。

除逆向进化假说外, 半酶理论是另一个基于生 命起源的异养说和中间代谢物可以稳定存的假说。 在半酶理论中, 代谢途径中的底物除了原始海洋中 的生命起源前复合物外, 还可以是从已经存在的代 谢途径中“漏出”的复合物。该假说的中心是代谢途 径中的一些反应最初是自发的非酶促反应, 后来其 底物逐渐消失，而其它代谢途径中的产物或者中间 产物在某一新产生的酶的作用下就得以替代原有 的物质, 非酶促反应就转变为酶促反应了。逆向进 化理论认为代谢途径的进化是由底物驱动的, 而底 物来自环境中的生命起源前复合物。Lazcano et al （1999）认为, 这种底物也可以是从已经存在的代 谢途径“漏出”的复合物, 因而提出了另一假说一 半酶理论。与逆向进化假说一样, 半酶理论也认为 原始海洋中最初有一套生命起源前复合物, 认同生 命异养起源说。半酶理论还说明了代谢途径中的酶 促反应是怎样由非酶促反应转变而来 (Lazcano et al，1999)。从已有代谢途径“漏出”的复合物是怎样 驱动代谢途径的进化呢? 参与组氨酸合成的 $\mathrm{G}$ 型 
谷氨酰胺酰胺转移酶（glutamine amidotransferase） - $\mathrm{HisH}$ 蛋白可以很好地说明这一点。经证实, 在 高氨浓度下没有 $\mathrm{HisH}$ 蛋白的参与, 也可以实现加 氮反应, 其中的氮原子来自 $\mathrm{NH}_{3}$ (Rieder et al, 1994)。因此, Lazcano 和 Miller（1996）认为该反 应最初由 $\mathrm{NH}_{3}$ 提供氮原子, 后来由于 $\mathrm{NH}_{3}$ 从原始海 洋中的消失和 HisH 蛋白的出现, 从其它代谢途径 “漏出”的谷氨酰胺就取代 $\mathrm{NH}_{3}$ 为反应提供氮原子。 也就是说, 生物合成途径的一些反应步骤在进化的 早期阶段没有酶参与, 是自发的, 当环境中一种底 物减少或消失需要其它代谢途径中生成的物质来 代替时, 非酶促反应就转变为酶促反应了。

与逆向进化假说相对的、一个鲜为人知的假说 是 1990 年由 F. Cordo'n 为解释分解代谢途径形成而 提出的向前发展模型。该学说认为原始的生命体靠 分解环境中的底物来获得能量。当环境中这种生命 体赖以生存的前体物缺乏时, 可以将其降解产物进 一步降解的生命体就获得了选择优势。早期生命体 靠将物质 A 降解成产物 1 获得能量, 当环境中物质 $\mathrm{A}$ 缺乏时, 能将产物 1 进一步降解成产物 2 从而获 得更多能量的生命体就占据了选择优势; 如此产物 2 又可以进一步降解, 使得该分解代谢途径得以进 一步延伸 (Cunchillos et al, 2007)。该假说与逆向 进化假说的一些理论前提是相同的, 如生命异养起 源说。但二者分别是为解释合成代谢途径和分解代 谢途径而提出的。

\section{2 酶的招募假说}

1975 年 Hartman 提出生命的自养起源说 (Hartman, 1975), 使逆向进化假说的前提面临质 疑。另外, 代谢途径中很多中间代谢物是不稳定的, 因此包含有这些中间代谢物的代谢途径则不能用 逆向进化假说来解释其进化 (Jensen et al, 1976)。 在这样的背景下, Ycas (1974) 提出了酶的招募假 说, 又称拼凑模型 (patchwork model)。该假说认 为代谢途径的进化可以通过 “招募”已有代谢途径的 酶, 从而形成一个由来自不同代谢途径、催化不同 反应的酶所组成的新的代谢途径 (Schmidt et al, 2003)。

该假说的前提是底物模糊性和原始细胞缺乏代 谢调节机制。底物模糊性表现在两个方面：1）原 始酶的底物专一性不强, 这可能是由于原始细胞酶 种类有限, 底物范围较宽可以使祖先细胞催化功能
最大化。2）现今酶的底物模糊性。现今很多蛋白/ 酶也表现有一定的底物模糊性, 例如 Miller \& Raines（2004）在细菌中发现糖激酶（sugar kinase） 有底物模糊性特点; Schofield et al (2005) 在细菌 Pyrococcus furiosus 中发现 3-脱氧-D 阿拉伯庚酮糖 7-磷酸-合成酶（3-deoxy-D-arabino-heptulosonate-7phosphate synthase, DAH7PS）也具有底物模糊性 特点, DAH7PS 不仅可以利用四碳磷酸单糖, 还可 以利用不同的五碳磷酸单糖。此外, Cove (1974) 认为原始细胞中尚未发展出代谢调节机制。酶的底 物模糊性使得祖先细胞的原始酶在一些特殊情况 下催化别的底物产生新的产物, 而代谢调节机制的 缺乏使得这种反应得以继续进行, 同时新产物也就 能够积累。如果新反应产物对细胞有用的话, 该细 胞就获得了某种选择优势 (Jensen et al，1976)。

支持酶的招募假说的一个强有力的证据是五氯 苯酚 (pentachlorophenol, PCP) 降解途径的形成。 $\mathrm{PCP}$ 是一种自然界原本不存在的杀虫剂, 所以在其 使用之前, 生物体不存在这种物质的降解途径。在 氯酚鞘氨醇单胞菌 (Sphingomonas chlorophenolica) 中分解 PCP 的代谢途径的第一个酶 (五氯苯酚脱氢 酶, pentachlorophenol hydroxylase) 和第三个酶 (2, 6一二氯氢醌双加氧酶, 2, 6-dichlorohydroquinone dioxygenase) 来自氯化苯酚（chlorinated phenol） 降解途径; 第二个酶（还原型脱卤素酶, reductive dehalogenase) 是由酪氨酸分解途径中的 MAA 异构 酶（maleylacetoacetate isomerase）进化而来的。由 此可见, PCP 分解途径是由来自两个代谢途径的酶 拼凑形成的 (Copley et al, 2000)。PCP 分解代谢途 径的发现表明, 代谢途径至今仍然在进化, 还表明 这一进化机制在今天的代谢途径形成过程依然发 挥作用, 似乎说明拼凑模型可能主要在代谢途经进 化的后期起重要作用。Nahum \& Riley（2001）在调 查大肠杆菌序列相关的蛋白群时发现的 20 对同源 酶中有 17 对有共同的反应化学和小分子的底物、 产物或辅助因子, 其中多数来自不同的代谢途径, 这可能是代谢途径进化过程中酶的招募的结果。除 了对同源酶的研究有越来越多的证据支持这一假 说外, 对酶特定结构域在代谢网络中的分布调查为 该假说在另一方面提供了佐证。Teichmann et al (2001) 在调查大肠杆菌小分子代谢时, 213 个结 构域家族中有 144 个成员分布于不同代谢途径; 另 外, Copley \& Bork（2000）还发现很多中心代谢途 
径中的酶具有 $\operatorname{TIM} \beta$ 桶或 TIM $\beta$ 桶样结构, 这也被 认为是代谢途径形成过程中酶的招募结果。

\section{3 多功能酶特化假说和整个代谢途径的复 制假说}

由于很多中间代谢物是不稳定的, Roy（1999） 认为代谢途径的进化不可能总是一步步进行, 也可 能是跳跃式逆向进化的, 由此提出多功能酶特化假 说一一代谢途径的进化可以通过募集低效的多功 能酶实现。这种低效的多功能酶可以催化代谢途径 中几步连续的反应, 在某些情况下可能会是一个代 谢途径的所有反应由一个原始的多功能酶催化, 这 个多功能酶经过基因复制和进一步特化为更为高 效的酶, 这样代谢途径就得以形成或延伸了。与酶 的招募假说相比，二者的共同点是都招募了已有代 谢途径中的酶, 并且都具有底物模糊性, 不同的是 在多功能酶的特化假说中这些被招募的酶能催化 多步反应，而在酶的招募假说中，这些被招募的酶 仅能催化一步反应。

多功能酶特化假说的理论基础是很多酶具有多 功能的属性。已知很多代谢中间物是不稳定的, 而 一个多功能酶可以催化连续多步反应将一个代谢 中间物转变成产物, 这就解决了逆向进化假说所不 能解决的代谢中间物不稳定问题。然而, 多功能酶 又是如何产生的呢? 在 Roy（1999）看来, 原始细 胞中的酶具有很弱的催化其它反应的活性。已发现 很多蛋白/酶具有不止一种催化活性, 如氨基甲酰磷 酸合成酶和脱氢奎宁酸合酶就是其中很好的例子。 氨基甲酰磷酸合成酶催化的四步连续反应中涉及 3 个不稳定的中间代谢物, 这一过程发生在氨基甲酰 磷酸合成酶的 4 个不同的活性部位（Raushel et al, 1998)。脱氢奎宁酸合酶则在同一个活性部位催化 五步连续的反应 (Carpenter et al, 1998)。另外, 很 多双功能酶的存在也是例证之一。大肠杆菌有 209 基因的产物有至少两种酶活性, 其中很多多功能酶 具有底物范围较宽的特点 (Light et al, 2004)。

如果一个代谢途径的所有酶是由一个原始多功 能酶经过复制和特化形成的, 那么整个代谢途径中 的酶或催化连续反应步骤的酶应该是同源酶。事实 上即使代谢途径中的酶源于同一个远古时期的多 功能酶祖先, 经过如此漫长的进化历程, 一级序列 上的相似性可能会很低, 然而其结构相似性却会保 留（Roy，1999)。该假说的支持证据难于发现的原
因在于结构信息的获得相对困难。由于多功能酶的 复制及特化会使代谢途径得以延伸，所以该假说可 以很好地解释代谢途径的延伸。但如果用于解释整 个代谢途径的形成则有一定的局限性，原因在于已 知的代谢途径都有很多的反应步骤, 且目前还没有 发现可以催化整个代谢途径所有反应的多功能酶。 如果一个多功能酶可以通过复制、特化形成新的代 谢途径，那么整个代谢途径复制后经过特化是否也 可以形成新的代谢途径呢? Schmidt et al (2003) 认 为这在进化过程中是完全可能的, 由此他们总结提 出了代谢途径重复假说, 认为新的代谢途径可以通 过已有的代谢途径作为一个整体进行复制, 然后转 换用途而形成。例如，色氨酸合成和组氨酸合成这 两个途径有几步连续的反应具有相似的反应机制, 且是由同源酶催化的, 很可能是早期这种代谢途径 重复的证据 (Gerlt et al, 2001; Schmidt et al., 2003)。

\section{4 从头创造假说}

从头创造假说首次由 Schmidt et al（2003）总结 提出, 该假说认为代谢途径无需采用已经存在的 酶, 可以自发形成, 即各个自发独立产生的酶可以 组合成一个代谢途径。例如独立起源的 tRNA 合成 酶, 后来参与了蛋白质的翻译、依赖于 tRNA 的转 酰胺基作用和非特异性的酰化作用三种不同的代 谢途径（Min et al，2002; Schmidt et al，2003）。

\section{5 总结和展望}

虽然有关代谢途径进化的假说已有 7 种, 但理 论体系完善且有比较强的证据支持的主要有“多功 能酶特化假说”、“逆向进化假说”以及“酶的招募假 说”。为什么会出现这样一个“众假说纷纭”, 而不能 统一为一个假说的局面呢? 我们认为可能的原因 如下：1）不同的代谢途径可能是在生物界不同的 进化阶段进化出来的, 所面临的前提条件和基础是 不一样的, 因而进化模式可能也就有所不同。如, 在生物进化的极早时期, 就较为符合“逆向进化假 说”和“向前发展假说”提出的情形，而不太可能是 “酶的招募”。因为要招募其它代谢途径的酶, 其前 提是被招募的这些代谢途径必需已经存在，因而这 只能是生物进化到一定阶段才能发生的进化模式。 2）随着生物的进化, 生物的代谢愈来愈复杂化, 各种代谢途径纵横交错, 互相协调以至于形成网络 化, 愈到后来发展出来的所谓某一代谢途径, 其实 
愈难脱离整个网络而独立成为一条单独的途径。因 此, 这时某一条代谢途径的进化实际上可能是整个 网络的进化问题, 而不能将其单独割裂出来进行其 进化的研究。基于这些分析, 我们认为若沿着生物 界进化发展的线路, 对处在不同进化地位的一系列 生物进行系统研究, 则可能有助于打破目前该领域 的研究停滞不前的局面, 利于对代谢途径的进化形 成整体的认识。正是基于这一认识, 本实验室对一 系列处在不同进化地位生物（包括原核生物的真细 菌和古细菌、原生生物的不同进化地位的代表生物 和不同地位的高等真核生物的代表) 的一些重要代 谢途径 (如糖酵解途径、光合作用途径和磷脂代谢 途径等) 进行了系统的调查分析。其中就有一些有 意思的重要发现, 如: 1) 贾第虫和真菌中的磷脂 酰丝氨酸合成酶、贾第虫与毛滴虫中的磷脂酰甘油 合成酶与植物中的对应直系同源物, 均来自于不同 真细菌的水平基因转移, 这符合“酶的招募假说”; 2) 而有些酶在从低等到高等生物中存在着底物专一 性不断特化的进化规律, 这似乎比较符合“多功能 酶的特化假说”（Tian，2008)。因此我们认为对于 同一个代谢途径而言，所涉及到的进化的机制则可

\section{参考文献:}

Belfaiza J, Parsot C, Martel A, de la Tour CB, Margarita D, Cohen GN, Saint-Girons I. 1986. Evolution in biosynthetic pathways: Two enzymes catalyzing consecutive steps in methionine biosynthesis originate from a common ancestor and possess a similar regulatory region [J] . Proc Natl Acad Sci, 83: 867-871.

Carpenter EP, Hawkins AR. 1998. Structure of dehydroquinate synthase reveals an active site capable of multistep catalysis [J]. Nature, 394: 299-301.

Cunchillos C, Lecointre G. 2007. Ordering events of biochemical evolution [J]. Biochimie, 89: 555-573.

Copley SD. 2000. Evolution of a metabolic pathway for degradation of a toxic xenobiotic: The patchwork approach [J]. Trends Biochem Sci, 25: 261-265.

Copley PR, Bork P. 2000. Homology among ( $\beta \alpha)_{8}$ barrels: Implications for the evolution of metabolic pathways [J] . J Mol Biol, 303: 627-640.

Cove DJ. 1974. Evolutionary significance of autogenous regulation [J]. Nature, 251-256.

Fani R, Liò P, Lazcano A. 1995. Molecular evolution of the histidine biosynthetic pathway [J]. J Mol Evol, 41: 760-774.

Fothergill-Gilmore LA, Michels PA. 1993. Evolution of glycolysis [J]. Prog Biophys Mol Biol, 59: 105-235.

Gerlt JA, Babbitt PC. 2001. Divergent evolution of enzymatic function: mechanistically diverse superfamilies and functionally distinct suprafamilies [J]. Annu Rev Biochem, 70: 209-246.

Hartman H. 1975. Speculations on the origin and evolution of metabolism [J]. J Mol Evol, 4: 359-370.

Horowitz NH. 1945. On the evolution of biochemical syntheses [J]. Proc
能都会有多种, 并且在不同的生物类群中其进化模 式也可能会有所不同。

另外, 迄今已有的有关代谢途径进化的研究无 一不是将注意力主要集中在酶的进化上, 然而完整 的代谢途径还包括除酶而外的调节因子、代谢中间 物以及代谢途径在细胞内的区室化定位等。这些因 素在代谢途径的演化过程中也应该起重要作用。因 此, 在研究代谢途径的进化时也应该考虑这些因 素。在这方面我们也进行了一些研究探讨。例如, 在单细胞的绿藻一一衣藻上, 我们在基因组和转录 组的水平上对其糖酵解途径在细胞内的区室定位 进行了全面系统的调查, 发现它与绝大多数真核生 物明显不同: 其大多数糖酵解酶仅定位于叶绿体 中, 因而胞质中缺乏完整的糖酵解途径（Sun, 2007)。这一研究结果不仅只是发现了糖酵解途径 的一种新的区室定位情形, 更重要的是对于揭示代 谢途径在不同生物类群中的演化具有重要意义。我 们有理由相信: 随着后基因组时代的到来，各种“组 学”数据的积累及生物信息学的飞速发展无疑会为 代谢途径乃至整个代谢网络的进化研究提供前所 未有的机遇。

Natl Acad Sci, 31: 153-157.

Jensen RA. 1976. Enzyme recruitment in evolution of new function [J]. Annu Rev Microbiol, 30: 409-425.

Lazcano A. 1999. On the origin of metabolic pathway [J]. J Mol Evol, 49: 424-431.

Lazcano A, Miller SL, Vents S. 1996. The origin and early evolution of life: Prebiotic chemistry, the pre-RNA world, and time $[\mathrm{J}]$. Cell, 85: 793-798.

Light S, Kraulis K. 2004. Network analysis of metabolic enzyme evolution in Escherichia coli [J]. BMC Bioinformatics, 5: 5.

Miller BG, Raines RT. 2004. Identifying latent enzyme activities: Substrate ambiguity within modern bacterial sugar kinases [J]. Biochemistry, 43 (21): 6387-6392.

Min B, Pelaschier JT, Graham DE, Tumbula-Hansen D, Söll D. 2002. Transfer RNA-dependent amino acid biosynthesis: An essential route to asparagine formation [J]. Proc Natl Acad Sci, 99: 2678-2683.

Nahum LA, Riley M. 2001. Divergence of function in sequence related groups of Escherichia coli proteins [J]. Genome Res, 11: 1375-1381.

Raushel FM, Thoden JB, Reinhart GD, Holden HM. 1998. Carbamoyl phosphate synthetase: A crooked path from substrates to products [J] . Curr Opin Chem Biol, 2: 624-632.

Rison SCG, Thornton JM. 2002. Pathway evolution, structurally speaking [J]. Curr Opin Struct Biol, 12: 374-382.

Rieder G, Merrick MJ, Castorph H, Kleiner D. 1994. Function of hisF and hisH gene products in histidine biosynthesis [J]. J Biol Chem, 269: 14386-14392.

Romano AH, Conway T. 1996. Evolution of carbohydrate metabolic 
pathways [J] . Res Microbiol, 147: 448-455.

Roy S. 1999. Multifunctional enzymes and evolution of biosynthetic pathways: retro-evolution by jumps [J]. Proteins, 37: 303-309.

Schmidt S, Sunyaev S, Bork P, Dandekar T. 2003. Metabollites: A helping hand for pathway evolution [J]. Trends in Biochem Sci, 28: 336-341.

Schofield LR, Anderson BF, Patchett ML, Norris GE, Jameson GB, Parker EJ. 2005. Substrate ambiguity and crystal structure of Pyrococcus furiosus 3-deoxy-D-arabino-heptulosonate-7-phosphate synthase: An ancestral 3-deoxyald-2-ulosonate-phosphate synthase[J]. Biochemistry, 44(36): 11950-62.

Teichmann SA, Rison SCG, Thornton JM, Riley M, Gough J, Chothia C. 2001. The evolution and structural anatomy of the small molecule metabolic pathways in Escherichia coli [J]. J Mol Biol, 311: 693-708.

Wilmanns M, Hyde CC, Davies DR, Kirschner K, Jansonius JN. 1991. Structural conservation in parallel beta/alpha-barrel enzymes that catalyze three sequential reactions in the pathway of tryptophan biosynthesis [J] . Biochemistry, 30: 9161-9169.

Ycas M. 1974. On earlier states of biochemical systems [J]. J Theor Biol, 44: 145-160.

Tian HF. 2008. Studies on glycerophospholipid biosynthesis pathway in several protists of important evolutionary status positions [D]. PH. D. thesis. Kunming Institute of Zoology, Chinese Academy of Sciences. [田海峰. 2008. 几类处在重要进化地位原生生物的甘油磷脂合成途 径的研究. 博士论文. 中国科学院昆明动物研究所.]

Sun GL. 2007. Studies on TIM genes and glycolytic pathway in several algae of important evolutionary status [D]. PH. D. thesis. Kunming Institute of Zoology, Chinese Academy of Sciences. [孙桂玲. 2007. 几 类处在关键进化地位藻类的 TIM 基因和糖酵解途径的研究. 博士 论文. 中国科学院昆明动物研究所.] 